\title{
On the Waiting Time of Arriving Aircrafts and the Capacity of Airports with Two Runways
}

\author{
N. Bäuerle $\stackrel{*}{,}$ O. Engelhardt-Funke and M. Kolonko ${ }^{\dagger}$
}

January 3, 2005

\footnotetext{
${ }^{*}$ Corresponding Author: Inst. for Mathematical Stochastics, University of Hannover, Welfengarten 1, D-30167 Hannover, Germany, email: baeuerle@stochastik.uni-hannover.de

${ }^{\dagger}$ Inst. for Mathematics, TU Clausthal, Erzstr.1, D-38678 Clausthal-Zellerfeld, Germany, email: kolonko@math.tu-clausthal.de
} 
Summary: In this paper we examine a model for the landing procedure of aircrafts at an airport. The characteristic feature here is that due to air turbulence the safety distance between two landing aircrafts depends on the types of these two machines. Hence, an efficient routing of the aircraft to two runways may reduce their waiting time.

First, we use M/SM/1 queues (with dependent service times) to model a single runway. We give the stability condition and a formula for the average waiting time of the aircrafts. Moreover, we derive easy to compute bounds on the waiting times by comparison to simpler queuing systems. In particular we study the effect of neglecting the dependency of the service times when using $\mathrm{M} / \mathrm{G} / 1$-models.

We then consider the case of two runways with a number of heuristic routing strategies such as coin flipping, type splitting, Round Robin and variants of the join-the-least-load rule. These strategies are analyzed and compared numerically with respect to the average delay they cause. It turns out that a certain modification of join-the-least-load gives the best results.

Key words: Queuing, Applied Probability, Air traffic control, Heuristic Routing 


\section{Introduction}

In this paper we examine the queuing process of aircrafts arriving at an airport and its implications for the capacity of the airport.

The particular feature of this process is the character of the safety distances between consecutive landing operations. An aircraft causes an air turbulence that endangers the stability of trailing aircrafts. The strength of the turbulence depends on the type (size, weight) of the leading aircraft. On the other hand, the type of the trailing aircraft determines its susceptibility to turbulence. Hence the required separation times between consecutive landing operations depend on the types of the two aircrafts involved.

This problem can be modeled as a special queuing system. The incoming planes are customers of different types and the service starts when the landing operation begins and it ends after the separation time has expired. Hence the service time depends also on the type of the next customer and we therefore do not have independent service times as it is assumed in standard queuing systems.

We are interested in stability results and bounds for the average waiting times of the aircrafts on a single runway as well as in the case where there are two runways available to which one arriving aircrafts have to be assigned.

We use the general assumption that the arrival times of aircrafts at the airport can be modeled by a Poisson process. This is in accordance with standard models in aircraft literature (see e.g. [5] and [8], Chapt. 8) and reflects the experience that in practice the flight schedule is disturbed by many independent external sources like weather or technical delays such that a Poisson model is sufficient for the type of average analysis intended here. The single runway problem can therefore be modeled as an M/SM/1 queuing model (see e.g. [6], [10],[11] and [12]).

For a single runway, we can use these results to derive the stability condition and to give an analytic formula for the expected stationary waiting time of the aircrafts which can be evaluated numerically. Moreover, simple bounds are derived by comparison to $M / G / 1$ and M/D/1 models. We examine the bias introduced when the dependencies of the service times are neglected and simple $\mathrm{M} / \mathrm{G} / 1$-models are used for the estimation of the average waiting time (which has been done for example in [5]). It is shown, that under heavy traffic this leads to a constant over- or underestimation of the average waiting time depending on the correlation of consecutive service times.

In the case of two runways we are interested in good heuristic routing strategies which make the average waiting time of the aircrafts small. Note that we do not address the problem of optimal assignment of aircrafts to runways here (see [1] for a qualitative result in that direction). Instead we only consider easy to implement heuristics like coin flipping, type splitting, Round Robin and variants of join-the-least-load. Type splitting is a randomized allocation of types to runways and allows to use the analytic results from the single runway case. It turns out that a certain modification of join-the-least-load performs quite well in our numerical example.

A possible extension of the model to include departing flights is sketched in the conclusion.

The scheduling of aircrafts has been treated before by different approaches, see e.g. [3] for an overview. The authors of [3] use a non-stochastic model in which they minimize waiting time related costs for a fixed set of planes by a mixed-integer approach. [4] presents an approach that tries to take the dynamics of the arrivals into account. A queuing approach is given in [5] where several strategies are compared and simulated. The authors use $M / D / n$ and $M / M / n$ queuing 
models that do not take into account the dependence of the service times. It is shown in Section 4 below, that estimates of waiting times based on these models may have a serious bias.

The paper is organized as follows. We first concentrate on the single server case. The model is described in Section 2 and a few results on the single server waiting times are collected in Section 3. In Section 4 we consider simple $\mathrm{M} / \mathrm{G} / 1$ and $\mathrm{M} / \mathrm{D} / 1$ models and compare the average waiting time of these models. The remaining sections deal with the case of two runways. In Section 5 we summarize some reasonable routing heuristics and compare their performance numerically in Section 6.

\section{The Model for a Single Runway}

\subsection{Arrivals}

We assume that the arrival times $\left(S_{n}\right)_{n>1}$ of the aircrafts form a renewal process with i.i.d. interarrival times $T_{n}:=S_{n}-S_{n-1}, n \geq 1$, and $S_{0}=T_{0}=0$. Let $\lambda:=\left(\mathbf{E} T_{1}\right)^{-1}>0$ denote the intensity of the arrival process and $\left(N_{t}\right)_{t \geq 0}$ the number of arrivals up to time $t$. Assume that the first aircraft arrives at time $T_{1}=S_{1}$.

Let $J$ be the finite set of possible types of aircrafts. We assume that a fraction $p_{j} \in(0,1)$ of all arrivals is of type $j \in J$ and that the types show up independently of each other and of the arrival process, i.e. if $J_{n}$ denotes the type of the $n$-th arriving aircraft, $n \geq 1$, then we assume

$$
P\left[J_{n}=i \mid T_{1}, \ldots, T_{n}, J_{1}, \ldots, J_{n-1}\right]=p_{i} .
$$

$p:=\left(p_{1}, \ldots, p_{|J|}\right)$ is the traffic mix and is assumed to be fixed.

Note, that if the arrival process is a Poisson process then it may be viewed as a superposition of independent Poisson processes $\left(N_{t, j}\right)_{t \geq 0}, j \in J$, each with intensity $\lambda_{j}:=p_{j} \lambda$.

\subsection{Separation times}

An aircraft of type $i \in J$ causes an air turbulence that forces any trailing aircraft of type $j \in J$ to keep a minimal distance expressed by the separation time $b(i, j)$. That means in particular, that the touch-down of the trailing aircraft must be separated by at least $b(i, j)$ time units from the touch-down of the leading aircraft.

We assume that the matrix $C:=(b(i, j))_{i, j \in J}$ of separation times is given.

\subsection{Service times}

If we model the aircrafts as customers, we obtain as service time of the $n$-th customer:

$$
B_{n}:=b\left(J_{n}, J_{n+1}\right) .
$$

Note that the service of the $n$-th aircraft starts with its touch-down and ends after time $b\left(J_{n}, J_{n+1}\right)$, where $J_{n+1}$ is the type of the next aircraft which may not even have arrived at that point of time.

From (2.1) it follows that the service times $\left(B_{n}\right)_{n \geq 1}$ are identically distributed and form a stationary process but they will in general not be independent. They may not even form a Markov 
chain. However, the interarrival times $\left(T_{n}\right)_{n \geq 1}$ and the service times $\left(B_{n}\right)_{n \geq 1}$ are independent from each other.

We define the traffic intensity on the runway as usual by

$$
\varrho:=\frac{\mathbf{E} B_{1}}{\mathbf{E} T_{1}}
$$

\section{$2.4 \quad$ Waiting times}

The waiting time of an aircraft is now defined as the time spent between its arrival at the airport until the beginning of the landing operation (touch-down). Then the waiting time $W_{n}$ of the $n$-th customer on the runway is given as usual recursively by

$$
\begin{aligned}
W_{1} & :=0 \\
W_{n+1} & :=\left[W_{n}+B_{n}-T_{n+1}\right]^{+}, \quad n \geq 1 .
\end{aligned}
$$

Our main interest is in the average waiting time

$$
\limsup _{N \rightarrow \infty} \frac{1}{N} \sum_{n=1}^{N} W_{n} .
$$

\subsection{Stability}

The sequence of waiting times $\left(W_{n}\right)_{n \geq 1}$ is said to be stable if there is a non-negative, real valued random variable $W_{\infty}$ such that

$$
\lim _{n \rightarrow \infty} W_{n}=W_{\infty}
$$

in distribution. Given the separation times, stability of the waiting times obviously depends on the arrival intensity $\lambda$. We are interested in the supremum $\bar{\lambda}$ over all $\lambda$ that allow stable waiting times. $\bar{\lambda}$ is called the ultimate capacity of the runway. It serves as an additional, simpler criterion in cases where we cannot determine the waiting times explicitly.

\section{Waiting Time and Capacity for a Single Runway}

We first collect a few results on the stationary waiting time in a queue with dependent service times from [9] and [12]. Let $D$ be the $|J| \times|J|$-matrix with entries $d(i, j):=p_{i} p_{j} b(i, j)$. Then

$$
\mathbf{E} B_{1}=\mathbf{E} b\left(J_{1}, J_{2}\right)=\sum_{i, j \in J} p_{i} p_{j} b(i, j)=\mathbf{1}^{T} D \mathbf{1}
$$

where $\mathbf{1}$ is the vector of dimension $|J|$, containing 1's only. Hence with

$$
\mathbf{E} T_{1}=\frac{1}{\lambda}
$$

we obtain

$$
\varrho=\lambda \mathbf{1}^{T} D \mathbf{1} .
$$

\subsection{Lemma}


a)

$$
\left(W_{n}\right)_{n \geq 0} \text { is stable } \quad \text { iff } \quad \varrho<1 .
$$

b) If $\varrho<1$, the sequence $\left(W_{n}\right)_{n \geq 0}$ is stochastically increasing, convergent in first mean and has, with $W_{\infty}$ as in $(2.6)$

$$
\begin{gathered}
W:=\mathbf{E} W_{\infty}<\infty \\
\lim _{N \rightarrow \infty} \frac{1}{N} \sum_{n=1}^{N} W_{n}=W \quad(\mathbf{P}-\text { a.s. })
\end{gathered}
$$

and

$$
\lim _{N \rightarrow \infty} \frac{1}{N} \sum_{n=1}^{N} \mathbf{E} W_{n}=W
$$

Proof : The proof follows essentially from Loynes [9]. To be more specific, let $U_{n}=B_{n}-$ $T_{n+1}, n \geq 1$, then

$$
W_{n+1}=\left[W_{n}+U_{n}\right]^{+}, \quad n \geq 1,
$$

where $\left(U_{n}\right)_{n \geq 1}$ is a stationary sequence and $\left(T_{n}\right)_{n \geq 1}$ and $\left(B_{n}\right)_{n \geq 1}$ are independent. From Loynes [9], Corollary 1 we obtain that $\varrho<1$ iff $\left(W_{n}\right)_{n \geq 0}$ is stable. Moreover we see from [9], Theorem 3 and Lemma1, that if $\varrho<1$, then there is a stationary sequence of a.e. finite, non-negative real valued random variables $M_{n}, n=\ldots-1,0,1, \ldots$ that forms a minimal solution of

$$
M_{n+1}=\left[M_{n}+U_{n}\right]^{+}, \quad n \geq 1 .
$$

It also follows that the distribution function $\mathbf{P}\left(W_{n} \leq t\right)$ converges for each $t \geq 0$ monotonically against $\mathbf{P}\left(M_{0} \leq t\right)$. Therefore, (2.6) holds with $W_{\infty}:=M_{0}$. As the sequence $\mathbf{P}\left(W_{n} \leq t\right)$ is monotonically decreasing, $W_{n}$ is stochastically increasing and convergence in the first mean follows. Hence also (3.7) follows. Lemma 3.2 below shows that we have $\mathbf{E} W_{\infty}<\infty$ under our condition $\varrho<1$. But then, the Strong Law of Large Numbers may be applied to the stationary sequence $M_{n}$ yielding

$$
\lim _{N \rightarrow \infty} \frac{1}{N} \sum_{n=1}^{N} M_{n}=\mathbf{E} M_{0}=\mathbf{E} W_{\infty} \quad(\mathbf{P}-\text { a.s. }) .
$$

It is also shown in Loynes [9], p. 503, that

$$
\lim _{n \rightarrow \infty}\left(M_{n}-W_{n}\right)=0 \quad(\mathbf{P}-\text { a.s. })
$$

hence

$$
\lim _{N \rightarrow \infty} \frac{1}{N} \sum_{n=1}^{N}\left(M_{n}-W_{n}\right)=0 \quad(\mathbf{P}-\text { a.s. }),
$$

which together with (3.8) proves (3.6).

From Lemma 3.1 a) we see that the ultimate capacity of the single runway is given by

$$
\bar{\lambda}:=1 / \mathbf{1}^{T} D \mathbf{1} .
$$


If the arrival process is a Poisson process, then a single runway forms an M/SM/1 queue where the types of services together with their duration form a semi-Markov process. These models have been intensively studied by M. Neuts e.g. in [13], and we can derive the following Lemma from [12].

\subsection{Lemma}

If the interarrival times are exponentially distributed with parameter $\lambda$ and if $\varrho<1$ then the expected stationary waiting time in the queue is given by

$$
W=\widetilde{B}-\mathbf{E} B_{1}+\frac{\lambda}{2(1-\varrho)}\left(\mathbf{E} B_{1}^{2}+2 \operatorname{cov}\left(B_{1}, B_{2}\right)\right)
$$

where $\widetilde{B}$ is the stationary mean service time of the first aircraft after the runway becomes empty.

Note that for independent service times $B_{1}, B_{2}$ we have $\widetilde{B}=\mathbf{E} B_{1}$ and $\operatorname{cov}\left(B_{1}, B_{2}\right)=0$, hence (3.10) reduces to the Pollazcek-Khintchine formula for the $\mathrm{M} / \mathrm{G} / 1$-model (see (4.7) below). Note also, that in (3.10) the only term depending on $\lambda$ is $\lambda /(2(1-\varrho))$. (3.10) is used in Theorems 4.2 and 4.3 below to compare $W$ to the waiting time of the corresponding $\mathrm{M} / \mathrm{G} / 1$ model.

Proof : The results in [12] are more general than needed here. The semi-Markov kernel for the service process is given by

$$
A_{i, j}(x):=\mathbf{P}\left(B_{1} \leq x, J_{2}=j \mid J_{1}=i\right)=p_{j} \mathbf{1}_{[0, x]}(b(i, j)) .
$$

Eq. (88) in [12] states that Little's law holds for the M/SM/1 system and eq. (70) gives a formula for the stationary mean number $L$ of customers in the system. Let $\alpha$ and $\alpha^{(2)}$ be the vectors with components

$$
\begin{aligned}
\alpha(i) & :=\mathbf{E}\left[B_{1} \mid J_{1}=i\right]=\sum_{j \in J} p_{j} b(i, j), \quad i \in J \\
\alpha^{(2)}(i) & :=\mathbf{E}\left[B_{1}^{2} \mid J_{1}=i\right]=\sum_{j \in J} p_{j} b^{2}(i, j), \quad i \in J,
\end{aligned}
$$

and let $p=\left(p_{i}\right)_{i \in J}$. Then we obtain from Eq. (70) in [12]

$$
\begin{aligned}
W & =\frac{L-\varrho}{\lambda} \\
& =g^{T} \alpha-\frac{\varrho}{\lambda}+\frac{1}{2 \lambda(1-\varrho)} \cdot\left[-2 \varrho^{2}+\lambda^{2}\left(p^{T} \alpha^{(2)}-2 p^{T} \hat{A}^{\prime}(0+) \alpha\right)\right]
\end{aligned}
$$

where the first derivative of the Laplace transform of the semi-Markov kernel $A$ is a $|J| \times|J|$ matrix with elements

$$
\hat{A}_{i j}^{\prime}(0+)=-\mathbf{E}\left[B_{1} \cdot \mathbf{1}_{\{j\}}\left(J_{2}\right) \mid J_{1}=i\right]=-b(i, j) p_{j} .
$$

$g=\left(g_{i}\right)_{i \in J}$ is the stationary distribution of the $J$-valued embedded Markov chain $\left(Y_{m}\right)_{m \geq 1}$ where $Y_{m}$ is the type of the aircraft that starts the $m$-th busy cycle on the runway. Note that in general this will be different from $p$ as this type has influenced the service time of the preceding aircraft and may therefore depend on the preceding busy cycle. Though we cannot 
give the explicit form of $g$, its existence is guaranteed whenever $\varrho \leq 1$ as noted in [12], see also [11]. Hence inserting (3.12) and (3.13) into (3.14) we obtain

$$
W=g^{T} \alpha-\frac{\varrho}{\lambda}+\frac{\lambda}{2(1-\varrho)}\left[-2\left(\frac{\varrho}{\lambda}\right)^{2}+\mathbf{E} B_{1}^{2}+2 \mathbf{E}\left(B_{1} B_{2}\right)\right]
$$

With $\widetilde{B}:=g^{T} \alpha=\sum_{i, j \in J} g_{i} b(i, j) p_{j}$ the assertion follows.

A numerical procedure for the approximation of $g$ as used in the proof above is given in Sect. 2.3 and 3.6 of [13]. In our case, let $G_{i, j}$ be the conditional probability, that given there is exactly one aircraft in the system and given the next one will be of type $i \in J$ that then at the first instance where the system becomes empty, the next type (that determined the service time just finished) will be of type $j \in J$. For $\varrho<1, G=\left(G_{i, j}\right)_{i, j \in J}$ is a $|J| \times|J|$-matrix that fulfills (cp. [13], Eq. (2.3.3))

$$
G=\sum_{n=0}^{\infty} A(n) G^{n}
$$

where $A(n)$ is another $|J| \times|J|$ - matrix with

$$
A(n)_{i, j}:=\mathrm{e}^{-\lambda b(i, j)} \frac{(\lambda b(i, j))^{n}}{n !} p_{j}
$$

for $n \geq 0$, i.e. $A(n)_{i, j}$ is just the probability that exactly $n$ aircrafts arrive during the service of an aircraft of type $i$ followed by one of type $j$. As is pointed out in [13], $G$ can be obtained from (3.17) by successive approximation. Finally, $g$ is the stationary distribution of $G$, which is guaranteed to exist for $\varrho<1 . g(i)$ is the stationary probability, that after an empty period, the next arrival will be of type $i \in J$. Using this approximation in Lemma $3.2, W=\mathbf{E} W_{\infty}$ can be evaluated numerically for each $\lambda$. This approach is used in the numerical example in Section 6 below.

In the next Section we use Lemma 3.2 to compare $W$ to results of other, simpler models.

\section{Waiting Times in Simplified Models}

In the aircraft literature (see [5] and [8]), often grossly simplified models are used. In particular, the waiting process is modeled as $M / G / 1$ or even $M / D / 1$ system, neglecting the dependencies between consecutive service times $B_{m}=b\left(J_{m}, J_{m+1}\right)$ and $B_{m+1}=b\left(J_{m+1}, J_{m+2}\right)$. However, as we will see, an $M / G / 1$-model may be a very bad approximation to the real situation.

In this Section we assume that the arrivals form a Poisson process with intensity $\lambda>0$. Let us first compare our model to two $M / D / 1$ systems. Let $\widetilde{W}$ be the average waiting time when the service times are given by the constant

$$
\widetilde{b}:=\mathbf{E} B_{1}=\sum_{i, j \in J} p_{i} p_{j} b(i, j)
$$

and let $\bar{W}$ be the average waiting time for constant service times

$$
\bar{b}:=\max \left\{b(i, j) \mid i, j \in J, p_{i} p_{j}>0\right\} .
$$

Note that by Lemma 3.1, stability in the case (4.1) holds iff $\varrho<1$, whereas for (4.2) we have to assume $\bar{b}<\lambda$. We have the following simple result.

\subsection{Theorem}


For $\varrho<1$ resp. $\bar{b}<\lambda$ we have

$$
\widetilde{W} \leq W \leq \bar{W}
$$

Proof : From (2.4) it follows for the $n$-th waiting time that

$$
W_{n}=\Phi_{n}\left(B_{1}, \ldots, B_{n-1}, T_{2}, \ldots, T_{n}\right)
$$

where $\left(b_{1}, \ldots, b_{n-1}\right) \mapsto \Phi_{n}\left(b_{1}, \ldots, b_{n-1}, t_{2}, \ldots, t_{n}\right)$ is an increasing, convex (and even supermodular) function, see also [2]. From Jensen's inequality we therefore obtain for all $n \geq 2$

$$
\begin{aligned}
\mathbf{E}\left[W_{n} \mid\right. & \left.T_{2}=t_{2}, \ldots, T_{n}=t_{n}\right]=\mathbf{E} \Phi\left(B_{1}, \ldots, B_{n-1}, t_{2}, \ldots, t_{n}\right) \\
& \geq \Phi\left(\mathbf{E} B_{1}, \ldots, \mathbf{E} B_{n-1}, t_{2}, \ldots, t_{n}\right) \\
& =\mathbf{E}\left[\widetilde{W}_{n} \mid T_{2}=t_{2}, \ldots, T_{n}=t_{n}\right]
\end{aligned}
$$

from which we conclude $\mathbf{E} W_{n} \geq \mathbf{E} \widetilde{W}_{n}$. Now, from Lemma 3.1 we have $W=\lim _{n \rightarrow \infty} \mathbf{E} W_{n}$ and $\widetilde{W}=\lim _{n \rightarrow \infty} \mathbf{E} \widetilde{W}_{n}$ which proves the first inequality. The second follows in a similar way from the monotonicity of $\Phi$.

Next let us consider a more realistic $M / G / 1$ model in which the i.i.d. service times $\widehat{B}_{n}, n \geq 1$, have the same marginal distribution as the original $B_{n}$, i.e.

$$
\mathbf{P}\left(\widehat{B}_{1} \leq x\right)=\mathbf{P}\left(B_{1} \leq x\right)=\sum_{i \in J} \sum_{j \in J} p_{i} p_{j} \mathbf{1}_{[0, x]}(b(i, j)) .
$$

Using this model means to simply drop the dependency between the service times. As $\mathbf{E} \widehat{B}_{1}=$ E $B_{1}$, stability holds in this model iff $\varrho<1$. For the corresponding mean stationary waiting time $\widehat{W}$ the classical Pollazcek-Khintchine formula holds:

$$
\widehat{W}=\frac{\lambda \mathbf{E}\left(\widehat{B}_{1}^{2}\right)}{2\left(1-\lambda \mathbf{E} \widehat{B}_{1}\right)} .
$$

The next Theorem gives upper and lower bounds for $W$ in terms of $\widehat{W}$. In addition to (4.2) we define

$$
\underline{b}:=\min \left\{b(i, j) \mid i, j \in J, p_{i} p_{j}>0\right\}
$$

\subsection{Theorem}

Let $\varrho<1$, then

$$
\underline{b}+C \leq W-\widehat{W} \leq \bar{b}+C,
$$

where

$$
C:=\frac{\lambda}{1-\varrho} \operatorname{cov}\left(B_{1}, B_{2}\right)-\mathbf{E} B_{1} .
$$

Note that the difference between upper and lower bounds in (4.8) is $\bar{b}-\underline{b}$, which is independent of the arrival rate $\lambda$. 
Proof : From Lemma 3.2 and (4.7) we have at once

$$
W=\widetilde{B}-\mathbf{E} B_{1}+\widehat{W}+\frac{\lambda}{1-\varrho} \operatorname{cov}\left(B_{1}, B_{2}\right)=\widetilde{B}+\widehat{W}+C .
$$

As $\widetilde{B}$ is a particular expected service time we have $\underline{b} \leq \widetilde{B} \leq \bar{b}$. From this the assertion follows.

The next Theorem shows, that depending on the correlation of the service times, the simple $\mathrm{M} / \mathrm{G} / 1$ model constantly over- or underestimates the true waiting times on the runways for heavy traffic and that the approximation error can get arbitrarily large.

\subsection{Theorem}
a) If
$\operatorname{cov}\left(B_{1}, B_{2}\right)>0$
then for $\varrho \uparrow 1$,
$W>\widehat{W}$ and $W-\widehat{W}$ tends to $\infty$.
b) If
$\operatorname{cov}\left(B_{1}, B_{2}\right)<0$
then for $\varrho \uparrow 1$,
$W<\widehat{W}$ and $W-\widehat{W}$ tends to $-\infty$.

Proof : Note that $\mathbf{E} B_{1}$ and $\operatorname{cov}\left(B_{1}, B_{2}\right)$ are independent of $\lambda$. Hence in (4.8), as $\varrho \uparrow 1$, we see that $C$ tends to $+\infty$ or $-\infty$ depending on the sign of $\operatorname{cov}\left(B_{1}, B_{2}\right)$, which proves the Theorem.

\section{Assigning Aircrafts to Two Runways}

After examining a single runway, we now turn to the full model where incoming aircrafts have to be assigned to one of two available runways, named $I$ and $I I$, by a routing policy $\delta$.

Now, $T_{1}, T_{2}, \ldots$ are the interarrival times of aircrafts arriving at that spot where the assignment decision is taken. $p=\left(p_{1}, \ldots, p_{|J|}\right)$ is the traffic mix of the arriving stream. This stream is split into two separate streams for the two runways by a routing policy. Let the interarrival times of the stream at runway $a \in\{I, I I\}$ be $T_{1}^{a}, T_{2}^{a}, \ldots$ We denote all local terms that refer to runway $a \in\{I, I I\}$ by an upper index $a$ as indicated by Figure 1 below, so e.g. on runway $I$ we have service times $B_{m}^{I}$, waiting times $W_{m}^{I}$ and $W_{\infty}^{I}$ (if it exists), $\lambda^{I}:=1 / \mathbf{E} T_{1}^{I}$ and traffic rate $\varrho^{I}$ if the interarrival times $T_{1}^{I}, T_{2}^{I}, \ldots$ are i.i.d. . Note that in $B_{m}^{a}, W_{m}^{a}$, etc the lower index $m$ is a local counter that counts the arrivals at runway $a$ only.

We assume here that the interarrival times $T_{1}, T_{2}, \ldots$ are i.i.d. and exponentially distributed, i.e. the arrival process at the airport is a Poisson process $\left(N_{t}\right)_{t \geq 0}$ with arrival rate $\lambda$.

In [1] the problem of minimizing the average waiting time of aircrafts being routed to two runways is formulated as a Markov decision program with discounted costs (it is mainly a technical question to extend these results to the average waiting times). Markovian routing policies $\delta$ are considered that assign aircrafts depending on the state of the runways and the type of the incoming aircraft. As it turned out, the structure of the model is quite involved, even simple monotonicity properties need not hold and there seems to be no simple analytic way to find optimal policies.

However, for practical purposes we are seeking policies that are simple to implement and are transparent to non-expert users. We therefore examine some simple types of policies first. 


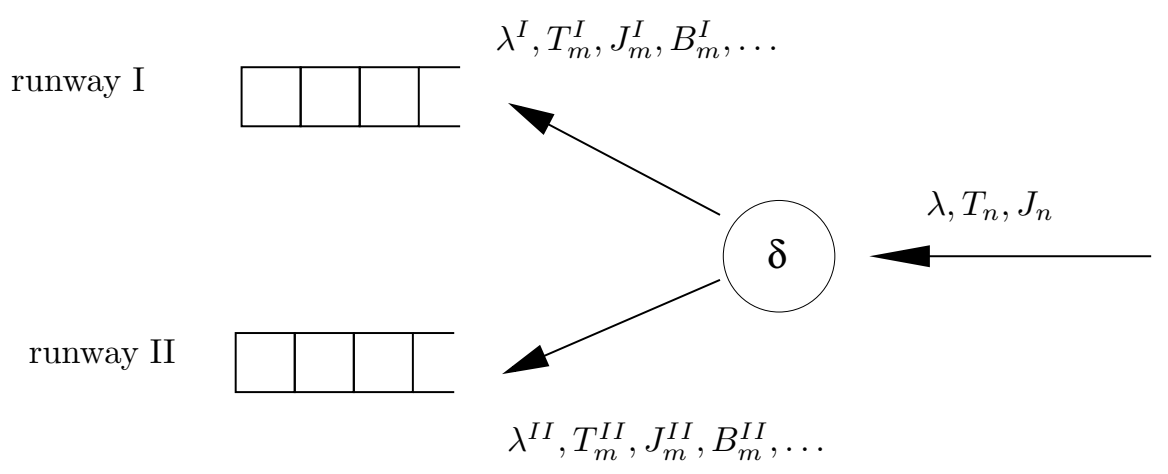

Figure 5.1: The incoming stream of aircrafts is split into two streams that arrive at the runways

\subsection{Random Split Policies}

First, we consider policies that only depend on the incoming type and 'split' the traffic mix between the two runways in a random way.

Let $\Delta$ denote the set of randomized decision rules $\delta=\left(\delta_{j}\right)_{j \in J}$ where

$$
\delta_{j} \in[0,1]
$$

is the probability for an aircraft of type $j$ to be assigned to runway $I$ and $1-\delta_{j}$ is the probability to be assigned to runway $I I, j \in J$. Let $A_{n}, n \geq 1$ denote the decision for the $n$-th arrival, i.e.

$$
\mathbf{P}\left(A_{n}=I \mid J_{n}=j\right)=\delta_{j}, \quad j \in J, n \geq 1 .
$$

Then the $A_{n}, n \geq 1$ are i.i.d.

The main technical advantage of restricting decision rules to $\Delta$ lies in the fact, that we obtain two independent queues at the runways. Applying decision rule $\delta \in \Delta$ splits the arrival process $\left(N_{t}\right)_{t \geq 0}$ into two independent Poisson arrival processes $\left(N_{t}^{I}\right)_{t \geq 0}$ and $\left(N_{t}^{I I}\right)_{t \geq 0}$ on the corresponding runways ("thinning"). Their intensities are

$$
\lambda^{I}:=\lambda \sum_{j \in J} p_{j} \delta_{j}=\lambda p^{T} \delta
$$

where $p:=\left(p_{j}\right)_{j \in J}$ and $\lambda^{I I}:=\lambda\left(1-p^{T} \delta\right)$. We assume that $p^{T} \delta \in(0,1)$, i.e. $\lambda^{I}>0, \lambda^{I I}>0$. Thus, the queues on each of the two runways are independent and behave as before in the single runway model. The probability that an aircraft arriving at runway $I$ is of type $i \in J$ is now given by

$$
p_{i}^{I}:=\frac{p_{i} \delta_{i}}{p^{T} \delta} \quad \text { and by } \quad p_{i}^{I I}:=\frac{p_{i}\left(1-\delta_{i}\right)}{p^{T}(\mathbf{1}-\delta)}
$$

for runway $I I . p^{I}$ and $p^{I I}$ are the two 'local traffic mixes'. Let $B_{m}^{a}$ denote the service time of the $m$-th plane on runway $a \in\{I, I I\}$, then we have

$$
\mathbf{E} B_{m}^{I}=\sum_{i, j \in J} p_{i}^{I} p_{j}^{I} b(i, j)=\frac{\delta^{T} D \delta}{\left(p^{T} \delta\right)^{2}} \quad \text { and } \quad \mathbf{E} B_{m}^{I I}=\frac{(\mathbf{1}-\delta)^{T} D(\mathbf{1}-\delta)}{\left(1-p^{T} \delta\right)^{2}}
$$

The (local) traffic intensities $\varrho^{I}$ and $\varrho^{I I}$ on runway $I$ and $I I$, respectively, are hence given by

$$
\varrho^{I}=\lambda \frac{\delta^{T} D \delta}{p^{T} \delta} \quad \text { and } \quad \varrho^{I I}=\lambda \frac{(\mathbf{1}-\delta)^{T} D(\mathbf{1}-\delta)}{1-p^{T} \delta} .
$$


Thus, the following Corollary to Lemma 3.1 gives a condition for the simultaneous stability on both runways.

\subsection{Corollary}

Under a routing policy $\delta=\left(\delta_{i}\right)_{i \in J} \in \Delta$, an arrival rate $\lambda$ leads to stability on both runways simultaneously iff

$$
\begin{aligned}
\lambda<\bar{\lambda}(\delta) & :=\min \left\{\frac{p^{T} \delta}{\delta^{T} D \delta}, \frac{p^{T}(1-\delta)}{(1-\delta)^{T} D(1-\delta)}\right\} \\
& =\left(\max \left\{p^{T} \delta \mathbf{E} B_{1}^{I},\left(\mathbf{1}-p^{T} \delta\right) \mathbf{E} B_{1}^{I I}\right\}\right)^{-1}
\end{aligned}
$$

Proof : (3.9) is equivalent to $\max \left\{\varrho^{I}, \varrho^{I I}\right\}<1$. Also, by $(5.3)$

$$
\frac{\delta^{T} p}{\delta^{T} D \delta}=\left(p^{T} \delta \mathbf{E} B_{1}^{I}\right)^{-1}
$$

and similarly for $a=I I$.

In accordance with Subsection 2.5, we call $\bar{\lambda}(\delta)$ as given in (5.5) the ultimate capacity of the runways under policy $\delta$. It may serve as a measure of performance for $\delta \in \Delta$. Numerical optimization techniques can be used to determine a strategy $\delta^{U l t C a p} \in \Delta$ that maximizes $\delta \mapsto \bar{\lambda}(\delta)$ over $\Delta$, see Section 6 below.

In the following Lemma, $W_{n}$ denotes the waiting time of the $n$-th incoming aircraft, irrespectively of the runway it is routed to, whereas $W_{m}^{a}$ is the waiting time of the $m$-th aircraft that lands on runway $a \in\{I, I I\}$ with expected stationary waiting time $W^{a}$ as given in Lemma 3.1.

We now show that the average of $W_{n}$ exists and is the weighted mean of the averages on the two runways.

\subsection{Lemma}

Assume that $\lambda<\bar{\lambda}(\delta)$. Then

$$
\begin{aligned}
\lim _{N \rightarrow \infty} \frac{1}{N} \sum_{n=1}^{N} W_{n} & =p^{T} \delta \cdot W^{I}+\left(1-p^{T} \delta\right) \cdot W^{I I} \quad(\mathbf{P}-\text { a.s. }) \\
& =: G(\delta, \lambda)
\end{aligned}
$$

Proof : 1. As we assume $\lambda^{a}>0, a \in\{I, I I\}$ we have $\lim _{k \rightarrow \infty} N_{S_{k}}^{a}=\infty$ ( a.s.), where $N_{S_{k}}^{a}$ is the number of customers that have arrived at runway $a$ up to the arrival time $S_{k}$ of the $k$-th customer in the system. But then from (3.6)

$$
\lim _{k \rightarrow \infty} \frac{1}{N_{S_{k}}^{a}} \sum_{m=1}^{N_{S_{k}}^{a}} W_{m}^{a}=W^{a} \quad \text { (a.s). }
$$


and, as $N_{S_{k}}^{a}=\sum_{n=1}^{k} \mathbf{1}_{\left[A_{n}=a\right]}$

$$
\lim _{k \rightarrow \infty} \frac{N_{S_{k}}^{a}}{k}=\mathbf{P}\left(A_{1}=a\right)=\left\{\begin{aligned}
p^{T} \delta & \text { for } a=I \\
1-p^{T} \delta & \text { for } a=I I
\end{aligned} \quad\right. \text { (a.s.). }
$$

2. From (5.7) and (5.8) we now obtain

$$
\begin{aligned}
\lim _{k \rightarrow \infty} \frac{1}{k} \sum_{n=1}^{k} W_{n} & =\lim _{k \rightarrow \infty} \frac{1}{k} \sum_{n=1}^{k}\left(W_{n} \mathbf{1}_{\{I\}}\left(A_{n}\right)+W_{n} \mathbf{1}_{\{I I\}}\left(A_{n}\right)\right) \\
& =\lim _{k \rightarrow \infty} \frac{1}{k}\left(\sum_{m=1}^{N_{S_{k}}^{I}} W_{m}^{I}+\sum_{m=1}^{N_{S_{k}}^{I I}} W_{m}^{I I}\right) \\
& =\lim _{k \rightarrow \infty} \frac{N_{S_{k}}^{I}}{k} \lim _{k \rightarrow \infty} \frac{1}{N_{S_{k}}^{I}} \sum_{m=1}^{N_{S_{k}}^{I}} W_{m}^{I}+\lim _{k \rightarrow \infty} \frac{N_{S_{k}}^{I I}}{k} \lim _{k \rightarrow \infty} \frac{1}{N_{S_{k}}^{I I}} \sum_{m=1}^{N_{S_{k}}^{I I}} W_{m}^{I I} \\
& =p^{T} \delta W^{I}+\left(1-p^{T} \delta\right) W^{I I} \quad \text { (a.s). }
\end{aligned}
$$

Bounds for $G(\delta, \lambda)$ can be obtained from Lemma 4.2. Using Lemma 3.2 and the approximation procedure described there, $G(\delta, \lambda)$ may be evaluated numerically for all $\delta \in \Delta$ and $\lambda<\bar{\lambda}(\delta)$. Numerical optimization may then be applied to approximate optimal policies $\delta^{\text {Split }}=\delta^{\text {Split }}(\lambda)$ that minimize $\delta \mapsto G(\delta, \lambda)$ under the constraint $\bar{\lambda}(\delta)>\lambda$. Further, a maximal $\lambda^{*}$ can be found such that the waiting time $G\left(\delta^{\text {Split }}\left(\lambda^{*}\right), \lambda^{*}\right)$ stays below a given threshold.

Maximizing the ultimate capacity $\delta \mapsto \bar{\lambda}(\delta)$ is much easier than minimizing $\delta \mapsto G(\delta, \lambda)$. The numerical results given below in Section 6 indicate that a strategy $\delta^{U l t C a p}$ that maximizes the ultimate capacity has waiting times $G\left(\delta^{U l t C a p}, \lambda\right)$ that are almost optimal under heavy traffic.

\section{$5.2 \quad$ Coin Flipping}

A particularly simple strategy is $\delta^{\text {Coin }} \in \Delta$ that flips a coin to assign an aircraft, i.e.

$$
\delta_{i}^{\text {Coin }} \equiv 1 / 2, \quad i \in J
$$

In this case we have for $i \in J$

$$
p_{i}^{I}=\frac{1 / 2 p_{i}}{\sum_{j \in J} 1 / 2 p_{j}}=p_{i}=p_{i}^{I I} .
$$

Also, $p^{T} \delta^{\text {Coin }}=1 / 2=1-p^{T} \delta^{\text {Coin }}$ and $\lambda^{I}=\lambda^{I I}=\lambda / 2$. Hence we see from (5.3) and (5.4) that

$$
\mathbf{E} B_{m}^{I}=\mathbf{E} B_{m}^{I I}=\mathbf{1}^{T} D \mathbf{1} \quad \text { and } \quad \varrho^{I}=\varrho^{I I}=\lambda \mathbf{1}^{T} D \mathbf{1} / 2
$$

This leads to the ultimate capacity

$$
\bar{\lambda}\left(\delta^{C o i n}\right)=\frac{2}{\mathbf{1}^{T} D \mathbf{1}} .
$$

Hence, strategy $\delta^{\text {Coin }}$ leads to an ultimate capacity that is twice the capacity of the single runway that was given in (3.9). This is of course the least one would expect from a good assignment. Simulation results below show, that $\delta^{\text {Coin }}$ is indeed a very bad choice for an assignment strategy in terms of average waiting times. 


\subsection{The Round Robin Policy}

Another very simple routing policy is the 'round robin' assignment $\delta^{R R o b i n}$ which assigns aircrafts nos. $1,3,5, \ldots$ to runway $I$ and nos. $2,4,6, \ldots$ to runway II.

Locally, on the two runways, we obviously have for $m=1,2, \ldots$

$$
\begin{aligned}
T_{m}^{I} & =T_{2(m-1)}+T_{2 m-1}, & & T_{m}^{I I}=T_{2 m-1}+T_{2 m} \quad \text { and } \\
J_{m}^{I} & =J_{2 m-1}, & & J_{m}^{I I}=J_{2 m}
\end{aligned}
$$

where $J_{n}$ is the type of the $n$-th aircraft arriving at the airport. Hence, the local arrival streams on the two runways are again renewal sequences with identical Erlang-2-distributions, but they are no longer independent of each other. Due to the i.i.d. -property of the types $\left(J_{n}\right)_{n \geq 1}$, the subsequences $\left(J_{m}^{I}\right)_{m \geq 1}$ and $\left(J_{m}^{I I}\right)_{m \geq 1}$ share the same distribution, in particular we have under $\delta^{R R o b i n}$

$$
p_{i}^{I}=p_{i}^{I I}=p_{i}, i \in J, \quad \text { and } \quad \mathbf{E} B_{m}^{I}=\mathbf{E} B_{m}^{I I}=\mathbf{1}^{T} D \mathbf{1}
$$

for all $m \geq 1$. As the interarrival times $T_{1}^{a}, T_{2}^{a}, \ldots$ are i.i.d. and independent of the stationary sequence $B_{1}^{a}, B_{2}^{a}, \ldots$, Lemma 3.1 still holds, but as they are no longer Poisson streams, Lemma 3.2 need not hold. From Lemma 3.1 and the above we have that stability on runway $a$ holds iff

$$
1>\varrho^{a}=\frac{\mathbf{E} B_{1}^{a}}{\mathbf{E} T_{1}^{a}}=\frac{\mathbf{E} B_{1}^{a}}{\mathbf{E}\left(T_{1}+T_{2}\right)}=\lambda \frac{\mathbf{1}^{T} D \mathbf{1}}{2} .
$$

Hence we have the ultimate capacity

$$
\bar{\lambda}\left(\delta^{R R o b i n}\right)=\frac{2}{\mathbf{1}^{T} D \mathbf{1}} .
$$

This is the same result as for coin flipping policy $\delta^{\text {Coin }}$, but as the simulation results below indicate, $\delta^{R R o b i n}$ is much better with respect to average waiting times.

Note that for the independent and exponentially distributed service times, $\delta_{R R o b i n}$ was shown to be optimal among all policies that do not take into account any information about the state of the runways (see [7]). Our numerical results in Section 6 indicate that this is not the case in our model as $\delta^{R R o b i n}$ is outperformed by $\delta^{U l t C a p}$ for heavy traffic.

\subsection{Join-the-least-load Strategies}

In [7] it was also shown that it is a good idea to try to balance the load of the two queues with respect to the available information on the state of the queues. A natural 'state' of runway $a \in$ $\{I, I I\}$ at the arrival time of the $n$-th aircraft would be the remaining waiting time (workload) $U_{n}^{a}$ and the type $\zeta_{n}^{a}$ of the last aircraft waiting on runway $a$. If the runway is empty, then $U_{n}^{a}$ is negative and gives the time that has elapsed since the touch-down of the last aircraft on that runway.

In [1], the present authors formulated a complete dynamic programming model based on these states. It is shown there, that if the available information is restricted to the states of the two runways and nothing is known about the arriving type, then the optimal policy has a join-theleast-load (JLL) property: if the types at the end of the two queues are identical, then any optimal policy assigns the $n$-th aircraft to runway $I$ if $U_{n}^{I}<U_{n}^{I I}$ and to runway $I I$ if $U_{n}^{I}>U_{n}^{I I}$.

Surprisingly, this need no longer hold, if the assignment policy is allowed to take the type of the arriving aircraft into account as is shown in [1] by a counterexample for finite horizon. 
In spite of this negative result, JLL is a useful principle for practical purposes. We define four different JLL-strategies. Let the information available for the assignment be given as

$$
\left(u^{I}, i^{I}, u^{I I}, i^{I I} ; k\right)
$$

where $u^{a}$ is the present workload on runway $a$ and $i^{a}$ the type of the last aircraft waiting on $a$ as described above. $k$ is the type of the presently arriving aircraft that has to be assigned to one of the runways.

We define the pure $J L L$-strategy $\delta^{J L L}$ by

$$
\delta^{J L L}\left(u^{I}, i^{I}, u^{I I}, i^{I I} ; k\right):=\left\{\begin{array}{cl}
I & \text { if } u^{I} \leq u^{I I} \\
I I & \text { if } u^{I}>u^{I I}
\end{array} .\right.
$$

The extended $J L L$-strategy $\delta^{J L L+}$ takes into account the type $k$ :

$$
\delta^{J L L+}\left(u^{I}, i^{I}, u^{I I}, i^{I I} ; k\right):=\left\{\begin{array}{cl}
I & \text { if } u^{I}+b\left(i^{I}, k\right) \leq u^{I I}+b\left(i^{I I}, k\right) \\
I I & \text { if } u^{I}+b\left(i^{I}, k\right)>u^{I I}+b\left(i^{I I}, k\right)
\end{array} .\right.
$$

The one-step-look-ahead JLL-strategy $\delta^{J L L 1 S t e p L A}$ looks one step ahead and takes into account the estimated effect of the next aircraft. Given that the present aircraft of type $k$ is routed to runway $I$, and that the next aircraft is routed according to $\delta^{J L L+}$, the expected additional waiting times on runway $I$ would be (for exponentially distributed interarrival times) :

$$
\begin{aligned}
& {\left[u^{I}+b\left(i^{I}, k\right)\right]^{+}} \\
& \quad+\sum_{l \in J} p_{l} \int_{0}^{\infty} \min \left\{\left[\left[u^{I}+b\left(i^{I}, k\right)\right]^{+}-t^{\prime}+b(k, l)\right]^{+},\left[u^{I I}-t^{\prime}+b\left(i^{I I}, l\right)\right]^{+}\right\} \lambda \mathrm{e}^{-\lambda t^{\prime}} d t^{\prime} .
\end{aligned}
$$

This value is replaced by a crude estimate:

$$
\operatorname{Load}(I):=\left[u^{I}+b\left(i^{I}, k\right)\right]^{+}+\min \left\{\left[\left[u^{I}+b\left(i^{I}, k\right)\right]^{+}-1 / \lambda+\alpha(k)\right]^{+},\left[u^{I I}-1 / \lambda+\alpha\left(i^{I I}\right)\right]^{+}\right\}
$$

where again $\alpha(i)=\sum_{j \in J} p_{j} b(i, j)$ for $i \in J$. Similarly, we put

$$
\operatorname{Load}(I I):=\left[u^{I I}+b\left(i^{I I}, k\right)\right]^{+}+\min \left\{\left[\left[u^{I I}+b\left(i^{I I}, k\right)\right]^{+}-1 / \lambda+\alpha(k)\right]^{+},\left[u^{I}-1 / \lambda+\alpha\left(i^{I}\right)\right]^{+}\right\} .
$$

Now, $\delta^{J L L 1 S t e p L A}\left(u^{i}, i^{I}, u^{I I}, i^{I I} ; k\right)$ routes to runway $I$ iff $\operatorname{Load}(I)<\operatorname{Load}(I I)$, i.e. iff

$$
\begin{array}{r}
{\left[u^{I}+b\left(i^{I}, k\right)\right]^{+}+\min \left\{\left[\left[u^{I}+b\left(i^{I}, k\right)\right]^{+}+\alpha(k)\right]^{+},\left[u^{I I}+\alpha\left(i^{I I}\right)\right]^{+}\right\}} \\
<\left[u^{I I}+b\left(i^{I I}, k\right)\right]^{+}+\min \left\{\left[\left[u^{I I}+b\left(i^{I I}, k\right)\right]^{+}+\alpha(k)\right]^{+},\left[u^{I}+\alpha\left(i^{I}\right)\right]^{+}\right\} .
\end{array}
$$

These three strategies turned out to be quite efficient in the simulation, see results below. Additionally, we introduce $\delta^{\text {Linear }}$ which is another extension of the JLL idea. Under $\delta^{\text {Linear }}$, an arriving aircraft of type $k$ is routed to runway $I$ iff

$$
A\left(i^{I}, i^{I I}, k\right) u^{I}+B\left(i^{I}, i^{I I}, k\right)<u^{I I} .
$$

Here, the linear coefficients $A, B$ may depend on the types $i^{I}, i^{I I}$ waiting at the end of the queues and the arriving type $k$. The values of $A(i, j, k), B(i, j, k), i, j, k \in\{1,2,3\}$ have to be chosen appropriately. In our simulation in the next Section we have determined these values by an intensive search using genetic algorithms and simulated annealing. 


\section{A Numerical Example}

To get an idea of how the strategies considered in the preceding Section might work in practice, we have evaluated their performance for a realistic example. We assumed that there are three different types of aircraft ('heavy', 'medium', 'light'). The matrix of separation times $C=$ $(b(i, j))_{i, j \in J}$ (in seconds) was extracted from [5]

$$
C=\left(\begin{array}{ccc}
96 & 120 & 144 \\
72 & 72 & 96 \\
72 & 72 & 72
\end{array}\right)
$$

The type mix $p:=(0.7,0.2,0.1)$ was also used in [5]. The resulting mean service time is $\mathbf{1}^{T} D \mathbf{1}=96$.

\subsection{Ultimate Capacity}

The coin flipping strategy has an ultimate capacity (see (5.10)) of

$$
\bar{\lambda}\left(\delta^{\text {Coin }}\right)=2 / \mathbf{1}^{T} D \mathbf{1}=2 / 96=0.02083 \quad \text { [aircrafts per second] }
$$

and corresponds to a capacity of 75 aircrafts per hour.

Maximizing the ultimate capacity $\bar{\lambda}(\delta)$ as defined in (5.5) over all $\delta \in \Delta=[0,1]^{3}$ numerically approximately yields

$$
\delta^{U l t C a p}:=(0.6965,0,0), \quad \bar{\lambda}\left(\delta^{U l t C a p}\right)=0.213166 \quad[1 / \mathrm{sec}]
$$

which corresponds to 76.74 aircrafts per hour. Here, $\delta^{U l t C a p}$ sends all medium and light aircrafts to runway $I I$ and $69.65 \%$ of the heavy ones to runway $I$.

\subsection{Average Waiting Time $G(\delta, \lambda)$}

Numerical evaluation and minimization of $\delta \rightarrow G(\delta, \lambda), \delta \in \Delta$ using the procedure of [13] described above was done with a standard software tool. Optimal strategies $\delta^{\text {Split }}$ and their waiting times for some values of $\lambda$ are given in the Table 1. For comparison, we also give the waiting times of the strategy $\delta^{\text {UltCap }}$ for the different values of $\lambda$. The values of $\lambda$ are chosen such that the corresponding traffic intensity $\hat{\varrho}$ on a single runway that processes the aircrafts with doubled speed is increasing to 0.9 . Here

$$
\hat{\varrho}:=\lambda \frac{\mathbf{1}^{T} D \mathbf{1}}{2}=48 \lambda .
$$

The average number of aircrafts arriving under $\lambda$ is given by $3600 \lambda$.

As can be seen, the optimal strategy $\delta^{\text {Split }}(\lambda)$ does not change very much with $\lambda$ and is hardly better than $\delta^{\text {UltCap }}$ which is much easier to obtain numerically.

\subsection{Simulation Results}

We compared the performance of different strategies by simulating their average waiting time $\lim _{N \rightarrow \infty} 1 / N \sum_{n=1}^{N} W_{n}$. For each value of $\lambda$ we took the average of $N=100000$ random arrivals and repeated this experiment 20 times. The resulting averages are shown in Figure 6.1 below 


\begin{tabular}{|l||c|c|c|c|}
\hline$\lambda$ & 0.0125 & 0.0145 & 0.0166 & 0.01875 \\
\hline \hline$\delta^{\text {Split }}(\lambda)$ & $(7.085,0.0,0.0)$ & $(7.05,0.0,0.0)$ & $(7.0,0.0,0.0)$ & $(7.0,0.0,0.0)$ \\
\hline$G\left(\delta^{\text {Split }}(\lambda), \lambda\right)$ & 66.506 & 99.877 & 165.325 & 342.216 \\
\hline$G\left(\delta^{\text {UltCap }}, \lambda\right)$ & 66.594 & 100.015 & 165.528 & 342.782 \\
\hline \hline$\hat{\varrho}$ & 0.6 & 0.7 & 0.8 & 0.9 \\
\hline aircraft $/ \mathrm{h}$ & 45 & 52.1 & 59.76 & 67.5 \\
\hline
\end{tabular}

Table 1: Some splitting strategies obtained by numerical optimization

for the coin flipping strategy $\delta^{\text {Coin }}$, the round robin strategy $\delta^{R R o b i n}$, the optimal splitting strategy $\delta^{\text {Split }}$ which was found to be optimal for $\lambda=0.01875$ (see Table 1 ), the strategy $\delta^{U l t C a p}$ maximizing the ultimate capacity and the join-the-least-load strategies $\delta^{J L L}, \delta^{J L L+}, \delta^{J L L 1 \text { StepLA }}$ and $\delta^{\text {Linear }}$. The strategy 'Linear' used the following $(3 \times 3 \times 3)$ coefficient matrices (see $\left.(5.19)\right)$, which were determined by heuristic optimization:

$$
\begin{aligned}
A(\text { 'heavy }, \cdot, \cdot): & =\left(\begin{array}{rrr}
2.074 & 0.164 & 1.316 \\
-1.487 & -2.408 & -2.550 \\
2.209 & -0.103 & -0.651
\end{array}\right) \quad A(\text { 'medium', }, \cdot, \cdot):=\left(\begin{array}{rrr}
0.578 & 0.908 & -1.153 \\
-1.966 & -3.500 & -3.255 \\
2.057 & 1.320 & 1.096
\end{array}\right) \\
A(\text { 'light' }, \cdot, \cdot): & =\left(\begin{array}{rrr}
1.317 & 1.579 & 3.827 \\
0.494 & -1.520 & 0.626 \\
2.595 & 1.526 & 1.108
\end{array}\right)
\end{aligned}
$$

and

$$
\begin{aligned}
B(\text { 'heavy, }, \cdot, \cdot) & :=\left(\begin{array}{lll}
0.825 & 0.178 & 0.052 \\
3.583 & 0.197 & 0.069 \\
5.228 & 0.027 & 0.044
\end{array}\right) \quad B(\text { 'medium', }, \cdot, \cdot):=\left(\begin{array}{ccc}
0.357 & 0.026 & 0.007 \\
1.198 & 0.168 & 0.012 \\
1.602 & 0.275 & 0.089
\end{array}\right) \\
B(\text { 'light', }, \cdot, \cdot) & :=\left(\begin{array}{lll}
0.081 & 1.148 & 0.371 \\
4.004 & 0.486 & 0.011 \\
4.522 & 0.015 & 0.045
\end{array}\right)
\end{aligned}
$$

The results in Figure 6.1 show that coin flipping is a very bad strategy. The strategies 'UltCap' and 'Split' are better, they can hardly be distinguished in the Figure, as was to be expected by the results of Table 1 . However, strategies that use some information on the system as the JLL-strategies do perform much better. Here, the strategies 'JLL' and 'RRobin' perform almost equally well. Both try to balance the workload on the two runways. Taking the present arrival into account in 'JLL+' improves the performance slightly. 'JLL1StepLA', that estimates the impact of the next arrival behaves poorly under light traffic and becomes increasingly better with more arrivals per hour. The best strategy is the linear extension 'Linear' of JLL.

This tendency is underlined by the results for heavy traffic which are given in Figure 6.2, where we dropped the strategies 'Split' and 'JLL' as they continued to perform almost identically to the simpler strategies 'UltCap' and 'RRobin', respectively. 


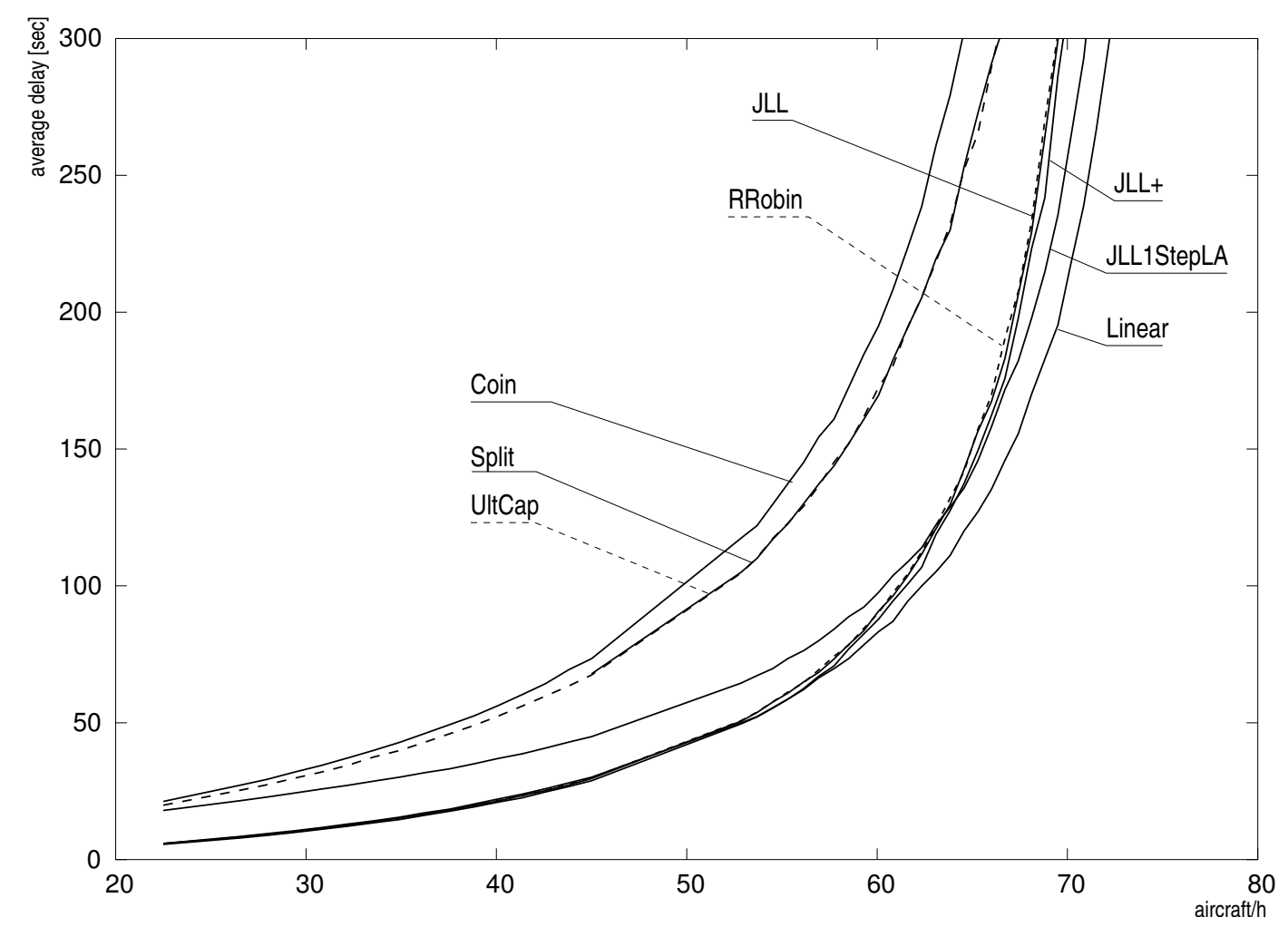

Figure 6.1: The simulated average waiting times of the strategies for light and medium traffic

Note that for strategies not in $\Delta$, $\hat{\varrho}>1$ may still lead to a stable system, as can be seen in Figure 6.2 for a number of aircraft/h of more than 75. Again the strategy 'Linear' is best. For increasing traffic intensity, the simple type split performed by 'UltCap' becomes relatively better and even beats 'RRobin' and 'JLL+' for values $\hat{\varrho}>0.98$ (corresponding to 74 aircraft/h). This indicates that splitting, i.e. sorting the types outperforms balancing the workloads for heavy traffic. The strategy 'Linear' combines both aspects and is clearly better than both.

Similar pictures were obtained for different values of the separation matrix and different type mixes.

\section{Conclusion}

Using results from $\mathrm{M} / \mathrm{SM} / 1$-queueing systems, we have derived the stability condition and the average waiting time for the single runway. We have shown that an approximation by simple queuing systems like $\mathrm{M} / \mathrm{G} / 1$ as it is used in the literature can be quite bad.

For the two runway case we studied several heuristic strategies analytically and experimentally. It turned out that under medium traffic simple type splitting or short-sighted load balancing are outperformed by more complex rules.

The model used in this paper may well be used to cover more general scenarios, e.g. if the runways are to be used for arriving and departing aircrafts. We then have to assume that the times at which aircrafts are ready to depart are sufficiently random such that the superposition of arrival times and departure times can still be modeled by a Poisson process. We can then extend the types of the aircrafts by an additional flag indicating whether this is an arrival or 


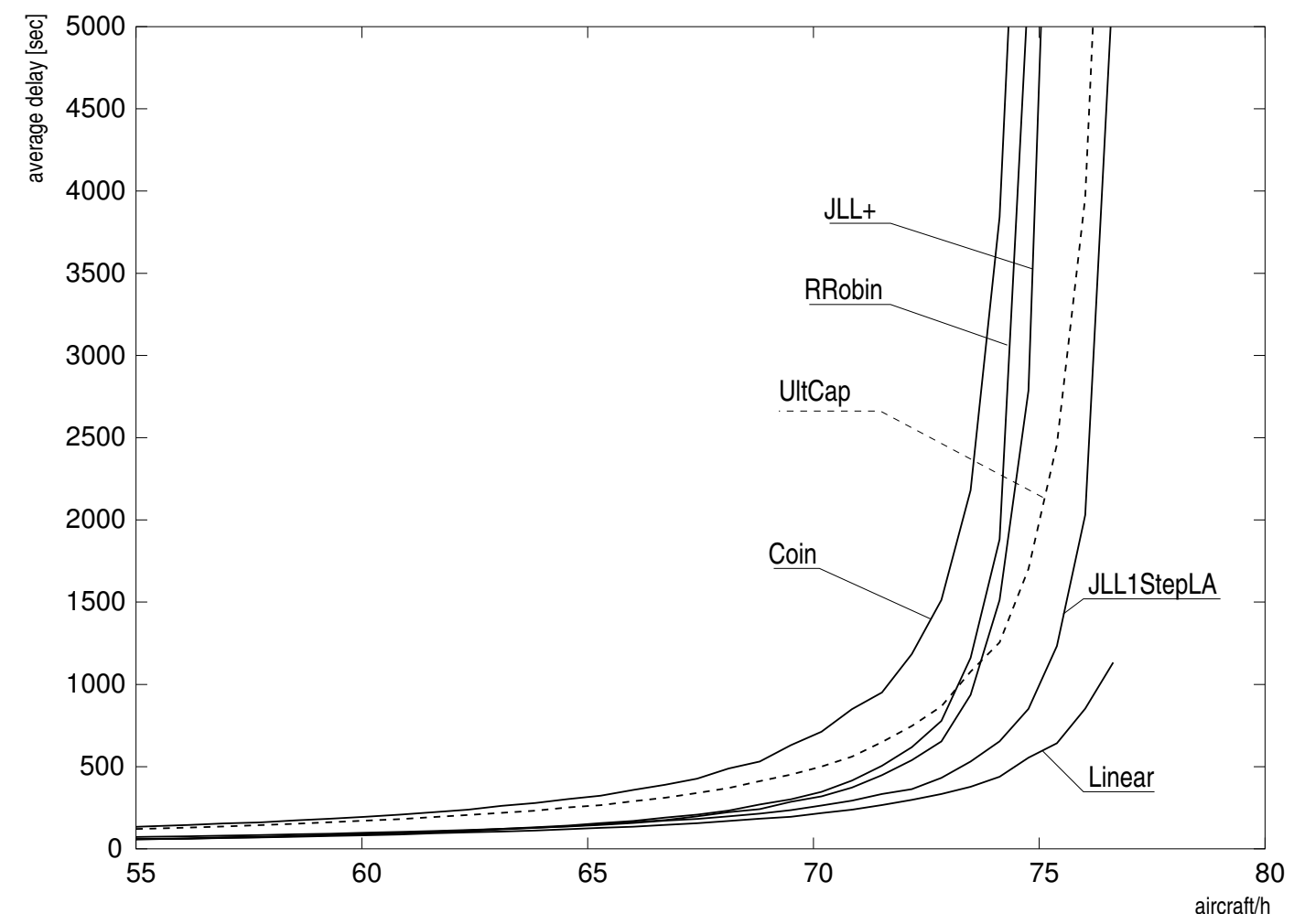

Figure 6.2: The simulated average waiting times of the strategies for heavy traffic.

a departure. The appropriate separation times have to be fixed and then the routing may be treated as above.

In the same way other features of flights that influence the required separation time may be included, like e.g. in which direction the runway is to be used or into what direction (air corridor) a departing aircraft will turn.

Our future work will concentrate on more complex routing decisions as it has been started in [1] and on different types of arrival patterns. Simulation should help to detect structures of optimal decisions which could be proven analytically.

\section{References}

[1] Bäuerle, N., O. Engelhardt-Funke And M. Kolonko (2004) Routing of Aircrafts to Two Runways: Monotonicity of Optimal Controls. Probability in the Engineering and Informational Sciences 18, 533-560.

[2] BäUerle, N. And U. Rieder (1997) Comparison results for Markov-modulated recursive models. Probability in the Engineering and Informational Sciences 11, 203-217.

[3] Beasley, J.E., M. Krishnamoorty, Y.M. Sharaiha and D. Abramson (2000) Scheduling Aircraft Landings - The Static Case. Transportation Science 34, p 180-197.

[4] Beasley, J.E., M. Krishnamoorty, Y.M. Sharaiha and D. Abramson (2000) The displacement problem and dynamically scheduling aircraft landings. Report available at http://mscmga.ms.ic.ac.uk/jeb/jeb.html. 
[5] Bolender, M.A. And G.L. Slater (2000) Evaluation of Scheduling Methods for Multiple Runways. Journal of Aircrafts 37, 410 - 416.

[6] Çinlar, E. (1967) Time dependence of queues with semi-Markovian services. J. Appl. Probab. 4, 356-364.

[7] Ephremides, A, P. Varaya and J. Walrand (1980)A Simple Dynamic Routing Problem. IEEE Transactions on Automatic Control AC-25, 690-693.

[8] Horonjeff, R. And F.X. McKelvey (1994) Planning and Design of Airports. McGrawHill, 4. ed., Boston.

[9] Loynes, R.M. (1962) The stability of a queue with non-independent inter-arrival and service times. Proc. Camb. Philos. Soc. 58, 497-520.

[10] Neuts, M.F. (1966) The Single Server Queue with Poisson Input and Semi-Markov Service Times. J. Appl. Prob. 3, 202-230.

[11] Neuts, M.F. (1976) Moment formulas for the Markov renewal branching process. Adv. Appl. Prob. 8, 690-711.

[12] Neuts, M.F. (1977) Some explicit formulas for the steady-state behaviour of the queue with semi-markovian service times. Adv. Appl. Prob. 9, 141-157.

[13] Neuts, M.F. (1989) Structured Stochastic Matrices of $M / G / 1$ Type and Their Applications Marcel Dekker, New York and Basel. 